\title{
Impacto do Sistema de Recompensa e do Acesso às Informações sobre o Desempenho Individual no Empowerment Psicológico e o seu Reflexo na Eficácia Gerencial de Empresa Multinacional
}

\section{Impact of the Reward System and of the Access to Information on Individual Performance in Psychological Empowerment and its Effect on the Management Effectiveness of a Multinational Company}

\author{
Sabrina do Nascimento \\ Doutoranda do Programa de Pós-Graduação em Administração - UNIVALI \\ Rua João Coan, 400 - Sala 408 - Bloco 1 - Biguaçu, SC - 88160-000 \\ sabnascimento@gmail.com \\ Ilse Maria Beuren \\ Doutora em Controladoria e Contabilidade - FEA/USP \\ Professora do Programa de Pós-Graduação em Ciências Contábeis da UFPR \\ Av. Pref. Lothário Meissner, 632 - Campus III - Jardim Botânico - Curitiba/PR - 80210-070 \\ ilse.beuren@gmail.com
}

\begin{abstract}
Resumo
O estudo objetiva verificar o impacto das variáveis sistema de recompensa e acesso às informações sobre o desempenho individual (antecedentes) no empowerment psicológico segundo o modelo de Spreitzer (1995), e o seu reflexo na eficácia gerencial (consequente) aferida pelo alcance das metas estabelecidas no sistema de recompensa, em uma empresa multinacional. Fundamenta-se o estudo no modelo conceitual de empowerment psicológico de Spreitzer (1995) e da sua rede estrutural utiliza duas variáveis antecedentes (sistema de recompensa e acesso às informações) como inputs e uma variável consequente (eficácia gerencial) como output. O constructo da pesquisa consubstancia-se de operacionalizações realizadas por Spreitzer (1995) e Lawler III (2003). Pesquisa descritiva com abordagem quantitativa foi realizada em empresa multinacional de origem americana, com uma unidade fabril instalada no Sul do Brasil. O questionário aplicado aos empregados desta unidade fabril foi adaptado de Spreitzer (1995), e contém 24 questões fechadas com escala Likert de sete pontos. $\mathrm{Na}$ análise dos dados aplicou-se a técnica estatística de regressão linear com a utilização do software Statistical Package for Social Sciences (SPSS). Os resultados da pesquisa evidenciam a presença das dimensões de competência, significado, impacto e autodeterminação do empowerment psicológico. A eficácia gerencial da empresa, aferida pelo alcance das metas estabelecidas no sistema de recompensa, não demonstrou relação significante com as dimensões do empowerment psicológico. Conclui-se que o sistema de recompensa e o acesso às informações sobre o desempenho individual impactam no empowerment psicológico e se refletem parcialmente na eficácia gerencial da empresa.
\end{abstract}

Palavras-chave: Sistema de Recompensa. Acesso às informações sobre desempenho individual. Empowerment Psicológico. Eficácia Gerencial. 


\begin{abstract}
The goal of this study is to investigate the impact of the variables reward system and access to information on individual performance (background) in the psychological empowerment according to the model of Spreitzer (1995), and its effect on managerial effectiveness (consequent) as measured by the achievement of goals set out in the reward system in a multinational company. The study is based on the psychological empowerment conceptual model of Spreitzer (1995) and its structure uses two independent variables (reward system and access to information) as input and a consequent variable (managerial effectiveness) as output. The construct of the research is consists of operationalizations done by Spreitzer (1995) and Lawler III (2003). A descriptive research with quantitative approach was performed on a multinational company of American origin, with a factory plant located in southern Brazil. The questionnaire sent to employees of this plant was adapted from Spreitzer (1995), and contains 24 multiple choice questions of seven points in Likert scale. In the data analysis, the statistical technique of linear regression was applied using the Statistical Package for Social Sciences (SPSS). The survey results show the presence of dimensions of the competence, meaning, impact and self-determination of psychological empowerment. The company's managerial effectiveness, as measured by the achievement of goals established in the reward system, showed no significant relationship with the psychological empowerment dimensions. It was conclude that the reward system and the access to information on individual performance impact on the psychological empowerment and are reflected partly in the company's managerial effectiveness.
\end{abstract}

Keywords: Reward System. Access to information on individual performance. Psychological Empowerment. Managerial Effectiveness.

\title{
1. Introdução
}

As organizações vêm buscando novas maneiras de se tornarem mais competitivas e eficientes nos seus processos. Uma das maneiras de conquistar esta eficiência e competitividade está na preocupação com seus empregados, sobretudo com sua remuneração. Acredita-se que uma melhor remuneração tende a gerar bem-estar, motivação para o alcance dos objetivos estratégicos e maior comprometimento com o negócio (NUNES, 2008).

Vieira e Pacheco (2004) mencionam que, por meio das demandas sociais, políticas e econômicas, as empresas perceberam que a competitividade dos seus produtos e serviços está diretamente ligada à produtividade e motivação das pessoas que as compõem. Nesta perspectiva, os gestores têm voltado suas atenções para o desenvolvimento de políticas de incentivo aos seus empregados no intuito de motivá-los.

Independente do nível de escolaridade e das informações que recebem, os empregados agem por estímulos. Assim, se receberem vantagens para realizar uma determinada atividade, então vão executá-la. As organizações buscam utilizar políticas de incentivo para estimular os indivíduos. Os estímulos podem ser na forma de desafios, delegação de direitos de decisão, de incentivos monetários ou de avaliação de desempenho individual (CICHELERO, 2004).

Os incentivos utilizados para influenciar os indivíduos podem ser de natureza negativa ou positiva. Os incentivos negativos ou punições advêm de um acontecimento que resulta na redução da satisfação ou anseio pessoal. Em contrapartida, os incentivos positivos ou prêmios são frutos de acontecimentos que resultam em maior satisfação pessoal, sendo que estes incentivos não podem ser obtidos sem a permanência ou entrada destes indivíduos na organização (ANTHONY; GOVINDARAJAN, 2002).

Existem várias maneiras de recompensar e incentivar os empregados, dentre elas Nascimento, Reginato e Lerner (2008) citam: treinamento, concessão de bolsas de estudo, 
promoção por meio da ocupação de uma função que requer maior responsabilidade, presentes aos colaboradores que se destacam no ambiente de trabalho, dentre outras. Griffin e Moorhead (2006, p. 166) afirmam que as recompensas "constituem-se, por exemplo, de muitos incentivos que as organizações oferecem aos empregados nos contratos psicológicos. Atendem também a algumas necessidades que os funcionários buscam satisfazer por meio da escolha de condutas de trabalho".

O sistema de recompensa adotado por uma empresa está imerso na complexidade do comportamento humano. Esta complexidade reside principalmente no grande número de variáveis que estão envolvidas, mesmo em situações relativamente simples (BERGAMINI, 1982, p. 31). Aduz ainda que as variáveis que afetam diretamente o comportamento humano podem ser dividas em individuais e ambientais. As variáveis individuais "dizem respeito a toda a bagagem inata, mais as experiências adquiridas ao longo das várias fases evolutivas, tais como infância, a adolescência e a fase adulta de cada um". As variáveis comportamentais "abrangem todos os possíveis eventos extrínsecos ao indivíduo, tais como grupo social, cultura, fatores do meio ambiente físico propriamente dito e muitíssimos outros".

As variáveis comportamentais estão diretamente relacionadas à motivação. Nascimento e Reginato (2009, p. 185) afirmam que "a motivação está relacionada aos objetivos e características pessoais. Assim, ela pode ser considerada um fator característico e intrínseco à essência do ser humano". Os autores destacam ainda que, "o desempenho dos funcionários na realização de suas tarefas possui relação com as teorias motivacionais, cujos propósitos são melhorar a compreensão das razões que estimulam pessoas a desempenharem melhor as suas atividades".

No caso específico da Teoria do Nível de Aspiração, abordada na Teoria Psicológica Motivacional, que é a Teoria Psicológica que fundamenta este estudo, está o empowerment em suas diversas aplicações. O empowerment representa os aspectos psicológicos dos empregados, que correspondem às necessidades individuais para obter uma sensação de controle em relação à tarefa desempenhada, sobretudo na experiência de trabalho destes indivíduos (SPREITZER, 2007).

Blanchard, Carlos e Randolph (2001, p. 15) destacam que o empowerment "é crucial para as empresas se tornarem competitivas no mundo dos negócios. [...] para as empresas serem bem sucedidas no novo mundo dos negócios, os membros da equipe devem sentir que trabalham em benefício próprio e que têm papéis importantes". O empowerment é "uma 'tecnologia de ponta' que fornece tanto a vantagem estratégica que as empresas estão procurando quanto a oportunidade que seus funcionários estão procurando" (BLANCHARD; CARLOS; RANDOLPH, 2001, p. 17).

A remuneração se reflete no comportamento e na motivação dos empregados detectados por meio do sistema de recompensa e do empowerment psicológico. $\mathrm{O}$ acesso às informações sobre o desempenho individual repercute nos níveis de desempenho da organização e no alcance da eficácia organizacional. Fortalece-se assim a ideia de que é necessário analisar o comportamento dos empregados para melhorar o desempenho organizacional e é nesta perspectiva que as empresas começam a utilizar novos conceitos e metodologias que têm como base teorias psicológicas motivacionais.

Nesse sentido, busca-se responder a seguinte questão norteadora da pesquisa: Qual o impacto das variáveis sistema de recompensa e acesso às informações sobre o desempenho individual (antecedentes) no empowerment psicológico segundo o modelo de Spreitzer (1995) e qual seu reflexo na eficácia gerencial (consequente) aferida pelo alcance das metas estabelecidas no sistema de recompensa? Assim o objetivo do estudo é verificar o impacto das variáveis sistema de recompensa e acesso às informações sobre o desempenho individual (antecedentes) no empowerment psicológico segundo o modelo de Spreitzer (1995), e o seu reflexo na eficácia gerencial (consequente) aferida pelo alcance das metas estabelecidas no 
sistema de recompensa, em uma empresa multinacional.

Este estudo demonstra sua relevância ao proporcionar novo conhecimento à contabilidade gerencial, por meio da interdisciplinaridade. Abordam-se as relações do sistema de recompensa e acesso às informações sobre o desempenho individual no empowerment psicológico e seu reflexo na eficácia gerencial, com base nas teorias psicológicas, sobretudo na Teoria do Nível de Aspiração, utilizando-se do modelo conceitual de Spreitzer (1995). O estudo justifica-se especialmente pela fase embrionária das pesquisas sobre tema, e espera-se que os resultados possam contribuir para o desenvolvimento de um instrumento de aferição da relação do sistema de recompensa e do acesso às informações sobre o desempenho individual com aspectos comportamentais dos empregados e seu impacto na eficácia gerencial.

\section{O Empowerment Psicológico}

O empowerment é definido por Conger e Kanungo (1988) como a relação de compartilhamento da autoridade e de recursos que implicam na motivação por meio do aumento da eficácia pessoal. Griffin e Moorheard (2006) asseveram que o empowerment é percebido de maneira mais ampla do que uma simples autonomia por parte do funcionário, ou seja, ele por si só promove a participação em uma variedade maior de áreas dentro da organização, incluindo mas não limitando o funcionário ao seu próprio ambiente de trabalho.

Siegall e Gardner (2000) afirmam que o conceito de empowerment está diretamente alinhado com a eficácia organizacional por meio da utilização inteligente dos recursos humanos da empresa. Zapelini (2008) assevera que o empowerment está presente na transferência de poder para um grupo social e na capacidade dos atores sociais realizarem autoavaliações de acordo com objetivos e procedimentos preestabelecidos por eles mesmos. De modo amplo, nos últimos anos as empresas têm procurado maneiras de ampliar e incentivar o empowerment no ambiente organizacional (GRIFFIN; MOORHEARD, 2006).

$\mathrm{O}$ empowerment psicológico corresponde a um conjunto de estados psicológicos que são necessários aos indivíduos para obterem uma sensação de controle em relação ao seu trabalho, em que, ao invés de concentrar seus esforços nas práticas gerenciais que compartilham o poder entre os empregados de todos os níveis hierárquicos, se concentra na experiência de trabalho destes indivíduos (SPREITZER, 2007). A perspectiva do empowerment psicológico está relacionada com as crenças pessoais dos empregados em relação a sua função junto à organização.

O modelo conceitual proposto por Spreitzer (1995) manifesta-se em quatro dimensões: competência, impacto, autodeterminação e significado. Estas dimensões atuam de forma combinada e conjunta, em que a ausência de apenas uma única dimensão terá impacto direto no empowerment psicológico (SPREITZER, 1995). A seguir, apresenta-se o entendimento de cada dimensão:

a) competência - é compreendida como a crença que o indivíduo possui em sua capacidade para realizar as atividades com habilidade (SPREITZER, 1995). Caracteriza-se também pelo desenvolvimento e aprendizagem no trabalho, com o intuito de aumentar a sensação de autodomínio (MENON, 2001);

b) impacto - reflete o grau de influência que um indivíduo possui no resultado final da realização de uma determinada tarefa (MATTHEUWS; DIAZ; COLE, 2003). Thomas e Velthouse (1990, p. 672) afirmam que esta dimensão "refere-se ao grau em que o comportamento é visto como 'fazendo a diferença' em termos de realização de uma tarefa, isto é, produzindo efeitos em seu ambiente de trabalho";

c) autodeterminação - aborda a motivação intrínseca dos indivíduos e sua relação de causalidade, que Thomas e Velthouse (1990) tratam como a dimensão de escolha em seu modelo. Ryan e Deci (2000, p. 54) afirmam que para um indivíduo estar 
motivado significa "ser movido para fazer alguma coisa". Quando um indivíduo está desmotivado, ele não deve sentir nenhum impulso ou inspiração para agir, já alguém motivado se demonstraria ativo em direção a uma determinada tarefa. Entretanto, a motivação não é um fenômeno individual ou único. Ela pode variar quanto ao tipo e nível de motivação, em que a distinção básica reside na motivação intrínseca e extrínseca (RYAN; DELCI, 2000). A motivação intrínseca se refere a fazer algo prazeroso ou agradável ao indivíduo. A motivação extrínseca está relacionada a fazer algo porque conduz a um resultado (RYAN; DELCI, 2000); e

d) significado - é entendida como o valor dado ao objetivo ou finalidade da tarefa desempenhada, julgada em relação aos próprios ideais ou normas do indivíduo (THOMAS; VELTHOUSE, 1990).

Essas dimensões do modelo conceitual proposto por Spreitzer (1995) consusbstanciam o presente estudo, conforme se descreve nos procedimentos metodológicos da pesquisa.

\section{Estudos Empíricos que utilizaram o Modelo de Spreitzer (1995)}

Procedeu-se a escolha de alguns estudos empíricos que versam sobre o empowerment psicológico dentre os 143 estudos anteriores encontrados na base de dados da Emerald e do Directory of Open Access Journals (DOAJ). Para a busca nas bases de dados, em 20 de maio de 2010, utilizou-se a terminologia psychological empowerment - Spreitzer. Na seleção dos estudos consideram-se os que mais se assemelham ao objetivo dessa pesquisa e os países em que as pesquisas foram realizadas.

Siegall e Gardner (2000) aplicaram um modelo multidimensional de empowerment psicológico e analisaram os fatores organizacionais que poderiam afetar os empregados internamente para não refinar ou não explorar a construção do empowerment na organização. Analisaram 203 empregados de uma fábrica da região centro-oeste dos Estados Unidos, sendo 159 horistas e 44 assalariados, que foram observados durante três dias no mês de setembro de 1997. Constataram-se a validade das dimensões propostas por Spreitzer (1995) no ambiente investigado quando associadas aos fatores contextuais.

Holdsworth e Cartwright (2003) exploraram a relação da satisfação e do stress no ambiente de trabalho com as quatro dimensões de empowerment psicológico, tendo como objetivos do estudo: a) analisar o empowerment psicológico dos empregados de um call center e comparar os resultados com os empregados de um ambiente de escritório tradicional; b) investigar uma escala de stress e satisfação no trabalho num call center e relacioná-la com a população em geral; e c) identificar se o empowerment psicológico está associado ao stress, satisfação profissional e à saúde mental e física dos empregados de um call center. A investigação foi realizada numa empresa de call center do noroeste do Reino Unido de prestação de serviços ao cliente e na divisão de segurança de uma grande organização internacional. Os resultados demonstraram que os empregados do call center possuem uma predisposição maior a situações de stress, menor satisfação profissional e índices mais baixos de saúde física e mental em relação a população em geral. Constataram que no empowerment psicológico, as dimensões de significado, impacto e autodeterminação estão diretamente relacionadas à satisfação profissional e não à saúde física e mental.

Dimitriades (2005) examinou a validade e confiabilidade do modelo de Spreitzer (1995) com o instrumento de Menon (1999) e as dimensões culturais de Hofstede (1999). Investigou o empowerment no contexto grego, aplicando-o em 154 alunos de duas instituições de ensino superior, na primavera de 2001. Na metodologia, optou pela utilização das quatro dimensões de Spreitzer (1995), com as dimensões de Menon (1999), compostas pelos fatores de controle, percepção de competência e a internalização do objetivo, associadas às dimensões culturais de Hofstede (1999). Os resultados obtidos corroboraram com a literatura Nascimento, S.; Beuren, I. M. 
na área, confirmando a visão de autodeterminação caracterizada pela percepção de impacto e competência com a internalização (significado). O nível de empowerment psicológico coadunou com estudos empíricos realizados em outros contextos culturais, como Canadá, Estados Unidos e Austrália. Observou ainda que os trabalhadores gregos são propensos a formalização (controle) no contexto investigado, mas para um maior grau de certeza da relação entre cultura e poder.

Bordin, Bartram e Casimir (2007) objetivaram explorar os antecedentes e os resultados do empowerment psicológico em uma grande multinacional americana do setor de tecnologia da informação que opera em Singapura. Formularam várias hipóteses para atingir o objetivo geral: a) o acesso às informações sobre a missão da organização irá se correlacionar positivamente com o empowerment psicológico; b) o acesso às informações sobre o desempenho irá se correlacionar positivamente com o empowerment psicológico; c) o acesso à informação sobre o sistema de recompensa da organização irá se correlacionar positivamente com o empowerment psicológico; d) a participação dos trabalhadores irá se correlacionar positivamente com o empowerment psicológico; e) o comprometimento organizacional irá se correlacionar positivamente com o empowerment psicológico; f) a satisfação no trabalho irá se correlacionar positivamente com o empowerment psicológico; g) a segurança no emprego irá se correlacionar positivamente com o empowerment psicológico; h) o apoio social supervisionado irá se correlacionar positivamente com o empowerment psicológico; i) a associação positiva entre o empowerment psicológico e a satisfação no trabalho será mais forte com os altos níveis de apoio social supervisionado do que com os baixos níveis de apoio social supervisionado. Os resultados revelaram que os fatores antecedentes de empowerment psicológico podem aumentar o comprometimento organizacional e a satisfação dos trabalhadores, e que o apoio social supervisionado é um fator determinante para os efeitos do empowerment na satisfação dos trabalhadores no contexto investigado.

Drake, Wong e Salter (2007) analisaram os impactos nos tipos específicos de feedback sobre o desempenho individual, com base no sistema de recompensa, na gestão dos empregados de nível operacional que afetam as dimensões de impacto, competência e autodeterminação, contempladas no modelo de empowerment psicológico proposto por Spreitzer (1995). O estudo foi realizado com 125 estudantes de uma instituição de ensino superior dos Estados Unidos. Os resultados demonstraram que o feedback e os sistemas de recompensa afetam de forma diferente as dimensões de empowerment. O retorno financeiro está associado positivamente à dimensão de impacto, entretanto quando relacionada às dimensões de autodeterminação e impacto apresentaram relação negativa. Perceberam ainda, que apenas os funcionários de níveis hierárquicos superiores apresentavam relação significativa entre a dimensão de impacto e a motivação.

Ghani, Hussin e Jusoff (2009) buscaram validar as dimensões cognitivas de empowerment psicológico apresentado por Spreitzer (1995) nas instituições de ensino superior da Malásia, com uma amostra composta por 312 docentes de 25 instituições de ensino superior privadas. $\mathrm{Na}$ análise dos dados, realizaram correlações, regressões, análise fatorial exploratória, utilizando a extração de componentes principais e rotação varimax para avaliar as cinco variáveis criadas com a contribuição de cada dimensão do empowerment psicológico. Os resultados revelaram que os antecedentes psicológicos possuem relação significativa com a dimensão de significado. Verificaram ainda que, o acesso à oportunidade de aprender e desenvolver são recursos preditores da dimensão de significado.

Patah et al. (2009) examinaram as influências do empowerment psicológico sobre a satisfação no trabalho global de recepcionistas da frente de trabalho em hotéis cinco estrelas na cidade de Lumpur da Malásia, com o objetivo de validar duas hipóteses: o empowerment psicológico do empregado influencia positivamente a satisfação no trabalho global; e o 
empowerment psicológico dos empregados impacta nos escores de satisfação geral do trabalho, que influenciam significativamente no perfil demográfico e de trabalho. A amostra constituiu-se de 210 respondentes distribuídos em 21 hotéis, sendo utilizados na análise dos dados o Alfa de Cronbach para aferir a confiabilidade do instrumento, estatística descritiva, Matriz de Correlação de Pearson das variáveis e ANOVA. Verificaram que a influência do empowerment psicológico dos empregados mostrou-se válido e significativo no estudo e relaciona-se com o aumento da satisfação global no trabalho. Os gestores possuem a necessidade de assegurar que os empregados satisfeitos demonstrem mais foco no desenvolvimento da criatividade, a fim de melhorar o seu nível de satisfação e desempenho, fornecendo assim qualidade na prestação de serviços aos clientes.

\section{Metodologia da Pesquisa}

Pesquisa descritiva com abordagem quantitativa foi realizada por meio de um estudo de caso. Esta pesquisa classifica-se como descritiva ao almejar a descrição do impacto das variáveis sistema de recompensa e acesso às informações sobre o desempenho individual (antecedentes) no empowerment psicológico de acordo com o modelo de Spreitzer (1995), e seu reflexo na variável eficácia gerencial (consequente) aferida pelo alcance das metas estabelecidas no sistema de recompensa.

No que concerne ao estudo de caso, conforme Yin (2003, p. 21), pesquisas dessa natureza devem "preservar as características holísticas e significativas dos eventos da vida real". Nesta estratégia "o pesquisador não tem controle sobre os eventos e variáveis, buscando aprender a totalidade de uma situação e, criticamente, descrever, compreender e interpretar a complexidade de um caso concreto" (MARTINS; THEÓPHILO, 2007, p. 61).

A empresa multinacional objeto de estudo tem unidades fabris localizadas em todos os continentes e distribuídas em 14 países. A empresa do grupo que foi selecionada para o estudo de caso possui 365 empregados na sua unidade fabril brasileira, estabelecida no Sul do Brasil, cuja denominação social não será revelada a fim de preservar seus objetivos e informações estratégicas. Esta empresa foi escolhida pela viabilidade de acesso aos dados relativos ao sistema de recompensa e avaliação de desempenho adotado na empresa para seus empregados. Também foi selecionada em decorrência da origem do seu capital, que é norteamericano. Presume-se que alguns aspectos culturais podem assemelhar-se ao estudo de Spreitzer (1995), realizado em empresas americanas.

A pesquisa fundamenta-se no modelo conceitual de empowerment psicológico proposto por Spreitzer (1995) e na rede estrutural do seu modelo, com a utilização de duas variáveis antecedentes - sistema de recompensa e acesso às informações (missão e performance) - como entradas (inputs) e a variável consequente - eficácia gerencial - como saída (outputs). Do modelo original não foram consideradas as seguintes variáveis: lócus de controle, autoestima, inovação, desejabilidade social e estabilidade ao longo do tempo. $\mathrm{O}$ constructo da pesquisa, que relaciona as hipóteses abarcadas pela problemática do estudo com suas variáveis e subvariáveis, consubstancia-se de operacionalizações realizadas nas pesquisas de Spreitzer (1995) e Lawler III (2003).

O Quadro 1 explicita o constructo da variável Sistema de recompensa, considerada um antecedente ao empowerment psicológico, conforme Spreitzer (1995). 
Quadro 1 - Constructo da Variável antecedente Sistema de recompensa

\begin{tabular}{|c|c|c|}
\hline Variáveis & Subvariáveis & Métricas das variáveis \\
\hline \multirow{10}{*}{ 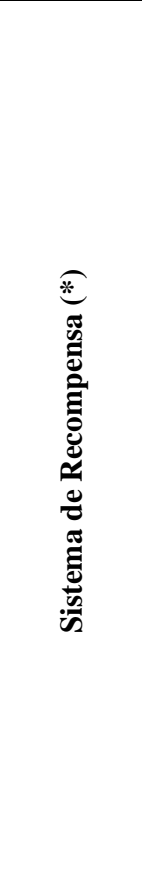 } & \multirow{2}{*}{ 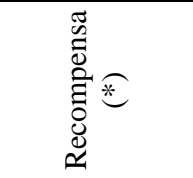 } & (*) Minha remuneração depende de como realizo meu trabalho \\
\hline & & $\begin{array}{l}\text { (*) Minha remuneração está diretamente relacionada com quanto bem realizo o meu } \\
\text { trabalho }\end{array}$ \\
\hline & \multirow{2}{*}{ 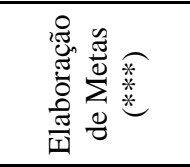 } & $\begin{array}{l}(* *) \text { Envolve os gestores na elaboração das metas para avaliação de desempenho } \\
\text { individual dos empregados }\end{array}$ \\
\hline & & $\begin{array}{l}\text { (**) Envolve os gestores na elaboração de suas próprias metas de avaliação de } \\
\text { desempenho }\end{array}$ \\
\hline & \multirow{3}{*}{ 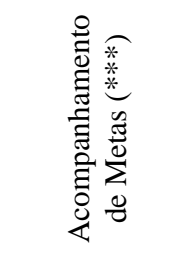 } & (**) Acompanha os empregados e motiva-os de acordo com o cumprimento das metas \\
\hline & & $\begin{array}{l}\text { (**) Satisfaz as necessidades dos empregados avaliados por meio do sistema de } \\
\text { recompensa }\end{array}$ \\
\hline & & $\begin{array}{l}\text { (**) Identifica os empregados com desempenho individual superior ou inferior a meta } \\
\text { elaborada }\end{array}$ \\
\hline & \multirow{3}{*}{ 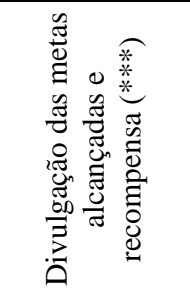 } & $\begin{array}{l}\text { (**) Divulga informações aos empregados sobre os prêmios de produtividade e } \\
\text { desempenho individual }\end{array}$ \\
\hline & & $\begin{array}{l}\text { (**) Divulga informações aos empregados sobre os prêmios de produtividade e } \\
\text { desempenho coletivo }\end{array}$ \\
\hline & & $\begin{array}{l}\text { (**) Divulga as informações sobre o desempenho global da empresa por meio das } \\
\text { ferramentas de tecnologia da informação que a organização dispõe }\end{array}$ \\
\hline
\end{tabular}

Fonte: adaptado de (*) Spreitzer (1995); (**) Lawler III (2003); e (***) elaboração própria.

No Quadro 1 observa-se a variável Sistema de recompensa, decomposta para fins deste estudo nas seguintes subvariáveis: recompensa; elaboração de metas; acompanhamento de metas; e divulgação das metas alcançadas e recompensa. Para cada subvariável foram atribuídas métricas, a fim de operacionalizar a variável na pesquisa empírica.

A subvariável Recompensa e as respectivas métricas foram extraídas do estudo de Spreitzer (1995), realizado com 393 gestores de uma empresa industrial e 128 empregados de uma seguradora, nos Estados Unidos.

Nas demais subvariáveis - Elaboração de metas, Acompanhamento de metas e Divulgação das metas alcançadas e recompensa -, as métricas foram agrupadas nessas três subvariáveis não existentes no modelo original de Spreitzer (1995). As respectivas métricas foram extraídas do estudo de Lawler III (2003), realizado em 17 grandes empresas americanas listadas pela Revista Fortune, em que foram entrevistados os gerentes de recursos humanos ou de recompensas e benefícios.

O Quadro 2 apresenta o constructo da variável Acesso às informações sobre desempenho individual, também considerada uma variável antecedente ao empowerment psicológico conforme Spreitzer (1995).

O Quadro 2 apresenta a variável antecedente Acesso às informações sobre o desempenho individual, decomposta nas subvariáveis: desempenho individual, acesso às informações da organização, fornecer medidas de desempenho, e feedback. Da mesma forma como na variável Sistema de recompensa, também nesta foram atribuídas métricas para cada subvariável.

A subvariável Desempenho individual e suas respectivas métricas foram extraídas da pesquisa realizada por Spreitzer (1995) em empresas norte-americanas. As outras subvariáveis - Acesso às informações da organização, Fornecer medidas de desempenho e Feedback - não constam no modelo de Spreitzer (1995). As métricas foram extraídas do estudo realizado por Lawler III (2003) e agrupadas nas três subvariáveis criadas. 
Quadro 2 - Constructo da Variável antecedente Acesso às informações sobre o desempenho individual

\begin{tabular}{|l|l|l|}
\hline Variáveis & Subvariáveis & \multicolumn{1}{|c|}{ Métricas das variáveis } \\
\hline \multirow{4}{*}{} & & (*) Acesso às informações estratégicas necessárias para desempenhar bem o meu \\
trabalho
\end{tabular}

Fonte: adaptado de (*) Spreitzer (1995); (**) Lawler III (2003); e (***) elaboração própria.

No Quadro 3 evidencia-se o constructo da variável Empowerment psicológico, concebida conforme o modelo de Spreitzer (1995).

Quadro 3 - Constructo da variável Empowerment psicológico

\begin{tabular}{|c|c|c|}
\hline Variáveis & Subvariáveis & Métricas das variáveis \\
\hline \multirow{16}{*}{ 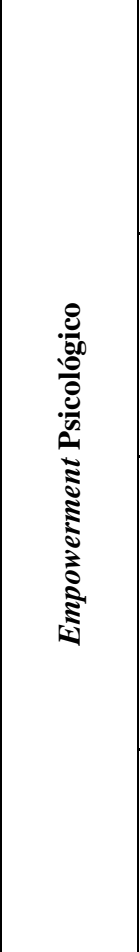 } & \multirow{4}{*}{ 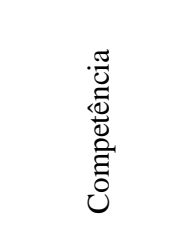 } & Estou confiante na minha capacidade de fazer o meu trabalho \\
\hline & & $\begin{array}{l}\text { Eu sou autoconfiante em relação às minhas capacidades para realizar as atividades do meu } \\
\text { trabalho }\end{array}$ \\
\hline & & Tenho domínio das competências necessárias para realizar o meu trabalho \\
\hline & & Meu trabalho está dentro do alcance de minhas habilidades \\
\hline & \multirow{4}{*}{$\begin{array}{l}\stackrel{̊}{0} \\
\text { : } \\
\stackrel{\Xi}{\Xi}\end{array}$} & Eu tenho influência significativa sobre o que acontece no meu departamento \\
\hline & & Eu tenho grande controle sobre o que acontece no meu departamento \\
\hline & & Minha influência é grande no que quer que aconteça no meu departamento \\
\hline & & Minha opinião conta na tomada de decisões do meu departamento \\
\hline & \multirow{4}{*}{ 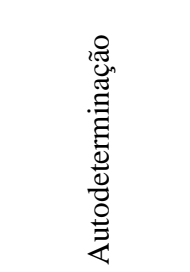 } & $\begin{array}{l}\text { Tenho oportunidade considerável para escolher com independência como fazer o meu } \\
\text { trabalho }\end{array}$ \\
\hline & & Tenho autonomia significativa na determinação de como eu faço o meu trabalho \\
\hline & & Eu posso decidir minha própria forma de fazer o meu trabalho \\
\hline & & Eu tenho chances de usar a iniciativa pessoal para realizar o meu trabalho \\
\hline & \multirow{4}{*}{ 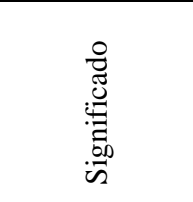 } & O trabalho que eu faço é importante para mim \\
\hline & & O trabalho que eu faço é significativo para mim \\
\hline & & Minhas atividades são pessoalmente significativas para mim \\
\hline & & Eu realmente me importo com o que eu faço no meu trabalho \\
\hline
\end{tabular}

Fonte: Spreitzer $(1995 ; 2010)$.

O Quadro 3 apresenta o construto do Empowerment psicológico, decomposto nas subvariáveis: competência, impacto, autodeterminação e significado. As métricas das subvariáveis foram extraídas da pesquisa realizada por Spreitzer (1995) e agrupadas de acordo

Nascimento, S.; Beuren, I. M. 
com Spreitzer (2010).

No Quadro 4 apresenta-se o constructo da variável Eeficácia gerencial, considerada uma variável consequente ao empowerment psicológico.

Quadro 4 - Constructo da variável consequente Eficácia gerencial

\begin{tabular}{|c|c|c|}
\hline Variável & Subvariáveis & Métricas das variáveis \\
\hline \multirow{16}{*}{ 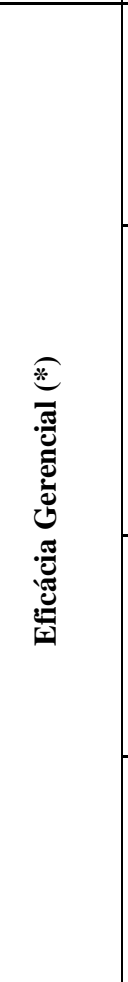 } & \multirow{4}{*}{ 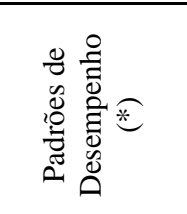 } & $(* *)$ Estabelece padrões (metas) individuais de desempenho \\
\hline & & $(* *)$ Existência de padrões de desempenho para equipes/departamentos \\
\hline & & (**) Existência de padrões de desempenho específicos para cada unidade de negócios \\
\hline & & $(* *)$ Existência de padrões de desempenho globais para a organização como um todo \\
\hline & \multirow{4}{*}{ 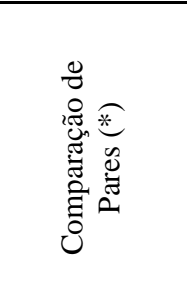 } & (**) Comparação de padrões (metas) individuais de desempenho previstos e realizados. \\
\hline & & $\begin{array}{l}\text { (**) Comparação de padrões de desempenho para equipes/departamentos previstos e } \\
\text { realizados }\end{array}$ \\
\hline & & $\begin{array}{l}\text { (**) Comparação de padrões de desempenho globais da unidade de negócios previstos e } \\
\text { realizados }\end{array}$ \\
\hline & & $\begin{array}{l}\text { (**) Comparação de padrões de desempenho globais da organização como um todo } \\
\text { previstos e realizados }\end{array}$ \\
\hline & \multirow{4}{*}{ 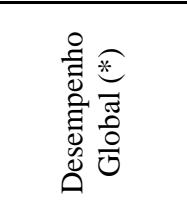 } & $(* * * *) \%$ de atingimento das diretrizes estabelecidas \\
\hline & & $(* * * *) \%$ de atingimento das metas estabelecidas \\
\hline & & $\begin{array}{l}\text { (****) Identificação dos benefícios (promoção, aumento salarial e bonificação) concedidos } \\
\text { aos trabalhadores }\end{array}$ \\
\hline & & $(* * * *) \%$ de crescimento do resultado econômico-financeiro da empresa \\
\hline & \multirow{4}{*}{ 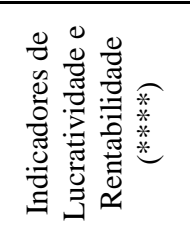 } & $(* * *)$ Taxa de retorno sobre o investimento (TRI) \\
\hline & & $(* * *)$ Taxa de retorno sobre o patrimônio líquido (TRPL) \\
\hline & & $(* * *)$ Margem operacional \\
\hline & & (***) Geração Operacional de caixa \\
\hline
\end{tabular}

Fonte: adaptado de (*) Spreitzer (1995); (**) Frezatti et al. (2009); (***) Assaf Neto (2001); e (****) elaboração própria.

O Quadro 4 apresenta a variável consequente Eficácia gerencial, decomposta nas subvariáveis - padrões de desempenho, comparação de pares e desempenho global agrupadas de acordo com o estudo de Spreitzer (1995). As subvariáveis Padrões de desempenho e Comparação por pares foram elaboradas com base em Frezatti et al. (2009), cuja obra trata da abordagem da contabilidade gerencial no contexto econômico, comportamental e sociológico. As métricas da subvariável Desempenho global são de elaboração própria.

Na subvariável Indicadores de lucratividade e rentabilidade, de Assaf Neto (2001) extraíram-se fórmulas para cálculo de indicadores de rentabilidade, especificamente Taxa de Retorno sobre o Investimento (TRI) e Taxa de Retorno sobre o Patrimônio Líquido (TRPL); e de lucratividade, particularmente Margem Operacional (MO) e Geração Operacional de Caixa (GOC). Os dados para calcular estes indicadores foram coletadas nos relatórios da administração de 2007 a 2010, disponíveis no site da Bolsa de Valores de Nova York (NYSE).

Os quatro constructos apresentados, que contemplam as variáveis Sistema de recompensa, Acesso às informações sobre desempenho individual, Empowerment psicológico e Eficácia gerencial, bem como suas respectivas subvariáveis, foram utilizados como subsídios para a elaboração do instrumento de pesquisa e a coleta de dados.

Aos empregados administrativos e operacionais da empresa encaminhou-se um questionário por correio eletrônico, entre os meses de agosto e outubro de 2010, para aferir a percepção desses em relação ao sistema de recompensa, acesso às informações sobre o desempenho individual e ao empowerment psicológico na empresa, em que se obteve 15 Impacto do Sistema de Recompensa e do Acesso às Informações sobre o Desempenho Individual no... 
respostas. O questionário constitui-se de 24 questões fechadas com escala likert de sete pontos, em que: $1=$ discordo muito fortemente; $2=$ discordo fortemente; $3=$ discordo; $4=$ neutro; $5=$ concordo; $6=$ concordo fortemente; e $7=$ concordo muito fortemente.

$\mathrm{Na}$ análise dos dados utilizou-se abordagem quantitativa. Para Hair Jr. et al. (2005), estudos desta natureza têm o intuito de fornecer uma visão panorâmica das variáveis observadas num determinado ponto do tempo e sintetizar seus resultados estatisticamente. Para relacionar as variáveis utilizaram-se os dados coletados junto aos empregados administrativos e operacionais por meio do instrumento de pesquisa e os dados extraídos da análise documental nos relatórios de administração da empresa do período de 2007 a 2010. Esses dados foram editados em planilha Excel, usada inicialmente para organizar os dados e calcular as frequências relativas das variáveis do estudo.

Após, realizou-se a aplicação da técnica estatística de análise multivariada de regressão linear, mediante a utilização do software Statistical Package for Social Sciences (SPSS) for Windows. Hair et al. (2005, p. 32) afirmam que esta técnica tem o intuito de "prever as mudanças na variável dependente como resposta a mudanças nas variáveis independentes". A interpretação mais direta da variável estatística de regressão, segundo Hair et al. (2005, p. 144-145), é a "determinação da importância relativa de cada variável independente na previsão da medida dependente (...). A análise de regressão fornece um meio de avaliar objetivamente a magnitude e a direção (positiva ou negativa) da relação de cada variável independente".

A utilização desta técnica justifica-se pela aplicabilidade em relação ao problema e ao objetivo dessa pesquisa, uma vez que busca analisar o impacto das variáveis independentes "sistema de recompensa", "acesso às informações sobre o desempenho individual" e "eficácia gerencial", em relação à variável dependente "empowerment psicológico". Buscou-se operacionalizar os objetivos específicos e realizar a validação das hipóteses da pesquisa para responder a indagação geral que norteia o estudo, tendo como ponto de partida a direção apontada pelo objetivo geral.

\section{Descrição e Análise dos Dados}

Esta seção apresenta a descrição e análise dos dados da pesquisa. A ênfase na análise está na relação das variáveis independentes - Sistema de recompensa, Acesso às informações sobre o desempenho individual e Eficácia gerencial - com a variável dependente Empowerment psicológico, e neste sentido elaboraram-se as hipótese a serem testadas:.

$\mathrm{H}_{1}-\mathrm{O}$ sistema de recompensa está positivamente relacionado com o empowerment psicológico segundo o modelo de Spreitzer (1995);

$\mathrm{H}_{2}-\mathrm{O}$ acesso às informações sobre o desempenho individual na empresa pesquisada está positivamente relacionado ao empowerment psicológico de acordo com o modelo proposto por Spreitzer (1995);

$\mathrm{H}_{3}-\mathrm{O}$ empowerment psicológico segundo o modelo de Spreitzer (1995) está positivamente relacionado com a eficácia gerencial na empresa estudada;

$\mathrm{H}_{4}-\mathrm{O}$ impacto das variáveis sistema de recompensa e acesso às informações sobre o desempenho individual (antecedentes) no empowerment psicológico está positivamente relacionado com a eficácia gerencial (consequente) na empresa analisada.

\subsection{Relação do sistema de recompensa com o empowerment psicológico do modelo de Spreitzer (1995)}

$\mathrm{O}$ resultado do teste da hipótese $\mathrm{H}_{1}-\mathrm{O}$ sistema de recompensa está positivamente 
relacionado com o empowerment psicológico segundo o modelo de Spreitzer (1995) - é apresentado na Tabela 1, que evidencia os coeficientes das dimensões do modelo de empowerment psicológico de Spreitzer (1995), que correspondem às variáveis dependentes associadas ao sistema de recompensa. Estes dados foram obtidos a partir da aplicação da técnica estatística de regressão linear, mediante a utilização do software Statistical Package for Social Sciences (SPSS) for Windows.

Tabela 1 - Coeficientes da variável sistema de recompensa relacionada ao empowerment psicológico por meio da análise de regressão

\begin{tabular}{l|c|c|c|c}
\hline $\begin{array}{l}\text { Dimensões do empowerment } \\
\text { psicológico }\end{array}$ & Coeficientes & Erro padrão & $\boldsymbol{t}$ & Sig. \\
\hline Competência & $-0,283$ & 0,213 & $-1,325$ & 0,210 \\
\hline Impacto & 0,170 & 0,275 & 0,620 & 0,547 \\
\hline Autodeterminação & $-0,442$ & 0,162 & $-0,656$ & 0,180 \\
\hline Significado & $-0,351$ & 0,174 & $-2,024$ & 0,066 \\
\hline
\end{tabular}

Os coeficientes de regressão das variáveis independentes apresentadas na Tabela 1 correspondem às dimensões de competência $(-0,283)$, impacto $(0,170)$, autodeterminação ($0,442)$ e significado $(-0,351)$, associadas à variável dependente sistema de recompensa. Hair et al. (2005) afirmam em relação ao coeficiente de regressão que demonstra o montante da variação na variável dependente quando associada a uma unidade de variável independente.

De acordo com Hair et al. (2005), o erro padrão é utilizado para demonstrar a significância dos resultados que procuram identificar se o coeficiente encontrado é de fato significativamente diferente de zero, incorrendo numa previsão mais confiável. Verifica-se na Tabela 10 que todas as dimensões do empowerment psicológico apresentaram valores diferentes de zero, demonstrando sua confiabilidade nas previsões.

O valor do $t$ de um coeficiente de regressão, segundo Hair et al. (2005, p. 133), é "o coeficiente dividido por seu erro padrão". Na Tabela 1 observam-se os valores das dimensões de competência $(-1,335)$, impacto $(0,620)$, autodeterminação $(-0,656)$ e significado $(-2,024)$.

O coeficiente de Significância (Sig.) das dimensões analisadas na Tabela 1, demonstra que as dimensões competência $(0,210)$, impacto $(0,547)$ e autodeterminação $(0,18)$ apresentaram significância superior a 0,10. Apenas a dimensão significado apresentou significância menor que 0,10 , demonstrando relação negativa com o empowerment psicológico.

Depreende-se que não é possível aceitar a hipótese $\mathrm{H}_{1}$, uma vez que a dimensão de significado apresentou relação negativa com a variável sistema de recompensa e as demais variáveis não possuem significância no modelo de regressão. Estes resultados diferem-se dos achados de Spreitzer (1995) em empresa industrial americana, bem como de Bordin, Bartram e Casimir (2007) que estudaram uma multinacional de Tecnologia da Informação em Singapura, quando detectaram relação significativa do sistema de recompensa com o empowerment psicológico.

\subsection{Relação do acesso às informações sobre o desempenho individual com o empowerment psicológico do modelo de Spreitzer (1995)}

A hipótese $\mathrm{H}_{2}-\mathrm{O}$ acesso às informações sobre o desempenho individual na empresa pesquisada está positivamente relacionado ao empowerment psicológico de acordo com o modelo proposto por Spreitzer (1995) - foi testada com aplicação da regressão linear nos dados obtidos por meio do questionário enviado aos empregados administrativos e operacionais, conforme demonstrado na Tabela 2. 
Tabela 2 - Coeficientes da variável acesso às informações sobre desempenho individual relacionada ao empowerment psicológico por meio da análise de regressão

\begin{tabular}{l|c|c|c|c}
\hline Dimensões do empowerment psicológico & Coeficientes & Erro padrão & $\boldsymbol{t}$ & Sig. \\
\hline Competência & 0,169 & 0,155 & 1,092 & 0,296 \\
\hline Impacto & 0,130 & 0,200 & 0,652 & 0,527 \\
\hline Significado & 0,267 & 0,126 & 2,118 & 0,56 \\
\hline Autodeterminação & 0,41 & 0,118 & 0,838 & 0,04 \\
\hline
\end{tabular}

$\mathrm{Na}$ Tabela 2, observa-se em relação aos coeficientes de regressão que esses apresentaram relação positiva com o acesso às informações sobre o desempenho individual em todas as dimensões de empowerment psicológico analisadas. Os coeficientes de erro padrão dos dados analisados apresentaram valores diferentes de zero nas dimensões de competência $(0,155)$, impacto $(0,130)$, significado $(0,267)$ e autodeterminação $(0,41)$.

O coeficiente de significância apresentou valores superiores a 0,10 nas dimensões competência, impacto e significado, mostrando-se assim não significantes em relação ao modelo proposto por Spreitzer (1995). Por outro lado, na dimensão autodeterminação atingiu valor inferior a 0,10 , evidenciando sua validade.

Infere-se do exposto que é possível aceitar parcialmente a hipótese $\mathrm{H}_{2}$, uma vez que a dimensão autodeterminação apresentou relação positiva $(0,41)$ e significante $(0,04)$ com o empowerment psicológico a partir do coeficiência de regressão. Esses achados coadunam em alguns pontos com os encontrados nas pesquisas de Spreitzer (1995) e Bordin, Bartram e Casimir (2007), que identificaram relação positiva entre as variáveis de acesso às informações sobre a missão da organização, acesso às informações sobre o desempenho da unidade e o empowerment psicológico.

No entanto, estes achados diferem dos resultados da pesquisa de Ghani, Hussin e Jusoff (2009), os quais constataram que a dimensão de impacto não tem relação significativa com o significado e a competência. Entretanto, tem baixa relação positiva com a autodeterminação, quando associadas às variáveis "acesso às informações"; "recursos"; e "apoio organizacional e oportunidade de aprender e desenvolver a confiança".

\subsection{Relação do empowerment psicológico com a eficácia gerencial}

$\mathrm{Na}$ hipótese $\mathrm{H}_{3}-\mathrm{O}$ empowerment psicológico segundo o modelo de Spreitzer (1995) está positivamente relacionado com a eficácia gerencial na empresa estudada -, buscou-se analisar esta relação a partir dos dados coletados nas demonstrações contábeis publicadas no site da Bolsa de Valores de Nova York (NYSE) (www.nyse.br). A Tabela 3 apresenta os dados coletados nas demonstrações contábeis da empresa analisada em milhares de reais.

Tabela 3 - Valores patrimoniais e de resultados para o cálculo da eficácia da empresa

\begin{tabular}{l|c|c|c|c}
\hline \multirow{2}{*}{ Itens } & \multicolumn{4}{|c}{ Valores acumulados } \\
\cline { 2 - 5 } & $\begin{array}{c}\mathbf{3}^{\mathbf{o}} \text { trimestre 2007 } \\
\text { acumulado }\end{array}$ & $\begin{array}{c}\mathbf{3}^{\mathbf{o}} \text { trimestre 2008 } \\
\text { acumulado }\end{array}$ & $\begin{array}{c}\mathbf{3}^{\circ} \text { trimestre 2009 } \\
\text { acumulado }\end{array}$ & $\begin{array}{c}\mathbf{3}^{\circ} \text { trimestre 2010 } \\
\text { acumulado }\end{array}$ \\
\hline Vendas líquidas & 772.253 & 837.331 & 639.695 & 669.737 \\
\hline Lucro operacional & 22.470 & 25.828 & $(51.808)$ & 51.693 \\
\hline Remuneração variável & - & - & 61.200 & 67.500 \\
\hline EBITDA & 15.692 & 26.437 & 18.469 & 27.652 \\
\hline Ativo total & 1.526 .977 & 1.561 .133 & 1.345 .149 & 1.260 .507 \\
\hline Patrimônio líquido & 599.671 & 613.420 & 422.613 & 420.800 \\
\hline
\end{tabular}

$\mathrm{Na}$ Tabela 3, têm-se as informações relacionadas às vendas líquidas, lucro operacional, EBITDA, ativo total e patrimônio líquido da empresa analisa, as quais foram utilizadas para realizar o cálculo dos indicadores de Lucratividade e Rentabilidade da empresa. A Tabela 4 apresenta o cálculo de indicadores de rentabilidade - Taxa de Retorno sobre o Investimento Nascimento, S.; Beuren, I. M. 
(TRI) e Taxa de Retorno sobre o Patrimônio Líquido (TRPL) - e indicadores de lucratividade - Margem Operacional (MO) e Geração Operacional de Caixa (GOC) -. Tais indicadores foram utilizados para demonstrar a eficácia gerencial da empresa estudada.

Tabela 4 - Cálculo dos indicadores para aferir a eficácia da empresa

\begin{tabular}{|c|c|c|c|c|c|c|c|c|c|}
\hline \multirow{3}{*}{ Indicadores } & \multirow{3}{*}{ Fórmula } & \multicolumn{8}{|c|}{ Valores acumulados } \\
\hline & & \multicolumn{2}{|c|}{$\begin{array}{c}3^{\circ} \text { trimestre } 2007 \\
\text { acumulado }\end{array}$} & \multicolumn{2}{|c|}{$\begin{array}{c}3^{\circ} \text { trimestre } 2008 \\
\text { acumulado }\end{array}$} & \multicolumn{2}{|c|}{$\begin{array}{c}3^{\circ} \text { trimestre } \\
2009 \text { acumulado }\end{array}$} & \multicolumn{2}{|c|}{$\begin{array}{c}3^{\circ} \text { trimestre } \\
2010 \text { acumulado }\end{array}$} \\
\hline & & \begin{tabular}{|c|} 
Índice \\
$(\%)$
\end{tabular} & $\begin{array}{c}\Delta \\
(06-07) \\
\end{array}$ & $\begin{array}{c}\text { Índice } \\
(\%)\end{array}$ & $\begin{array}{c}\Delta \\
(07-08)\end{array}$ & \begin{tabular}{|c|} 
Índice \\
$(\%)$
\end{tabular} & $\begin{array}{c}\Delta \\
(07-09)\end{array}$ & \begin{tabular}{|c|} 
Índice \\
$(\%)$
\end{tabular} & $\begin{array}{c}\Delta \\
(07-10) \\
\end{array}$ \\
\hline \multirow[t]{2}{*}{ Lucratividade } & $\mathrm{MO}=\frac{\text { Lucro op. líquido }}{\text { Vendas líquidas }}$ & 2,90 & - & 3,08 & 0,06 & $-8,09$ & $-1,78$ & 7,71 & 1,65 \\
\hline & $\begin{array}{c}\mathrm{GOC}=\text { EBITDA } \\
\text { Vendas líquidas }\end{array}$ & 2,03 & - & 3,15 & 0,55 & 2,88 & 0,41 & 4,12 & 1,02 \\
\hline \multirow{2}{*}{ Rentabilidade } & TRI $=\frac{\text { Lucro op. líquido }}{\text { Ativo total }}$ & 1,47 & - & 1,65 & 0,12 & $-3,85$ & $-1,61$ & 4,10 & 1,78 \\
\hline & $\begin{array}{l}\text { TRPL }=\text { Lucro op. } \\
\text { Patrimônio líquido }\end{array}$ & 3,74 & - & 4,21 & 0,12 & $-12,25$ & $-2,27$ & 12,28 & 2,280 \\
\hline
\end{tabular}

Em relação às informações dispostas na Tabela 4, destaca-se que a variação percentual entre os indicadores de lucratividade e de rentabilidade de cada período foram calculados com base nos valores do $3^{\circ}$ trimestre de cada ano. Os índices calculados relativos aos valores acumulados até o $3^{\circ}$ trimestre de 2009 apresentaram variações negativas, provavelmente em decorrência de reflexos da crise mundial.

No que concerne às variações dos trimestres analisados, percebe-se uma evolução crescente nos indicadores de lucratividade do $3^{\circ}$ trimestre de 2007 em relação ao $3^{\circ}$ trimestre de 2010. Os indicadores de rentabilidade apresentaram comportamento semelhante ao demonstrar a evolução positiva em suas variações. Contata-se ainda no $3^{\circ}$ trimestre de 2007 em relação ao $3^{\circ}$ trimestre de 2009 a presença de variações negativas.

A partir do comportamento das variações trimestrais da empresa analisada nos últimos quatro anos, nota-se que esta possui eficácia gerencial em sua gestão, uma vez que seus indicadores de lucratividade e rentabilidade apresentam evolução ao longo do tempo, com exceção do ano de 2009, que sofreu os reflexos da crise mundial. Estes achados coadunam com as respostas dos gestores entrevistados, quando asseguram o crescimento contínuo da empresa multinacional analisada, mesmo sem precisar/indicar estes percentuais de crescimento.

A tendência observada nos dados coletados por meio do instrumento de pesquisa e validados pelos indicadores de lucratividade e rentabilidade, calculados a partir das informações extraídas das demonstrações contábeis e do relatório da administração da empresa, remete a aceitar a hipótese $\mathrm{H}_{3}$, que relaciona o empowerment psicológico com a eficácia gerencial. Os resultados encontrados corroboram de certo modo os achados de Spreitzer (1995), que constatou a presença de uma relação significativa entre a eficácia gerencial, o empowerment psicológico e o comportamento inovativo.

\subsection{Impacto das variáveis antecedentes no empowerment psicológico e a relação com a eficácia gerencial}

Neste item realiza-se a análise da relação das variáveis antecedentes - sistema de recompensa e acesso às informações sobre o desempenho individual - com o empowerment psicológico segundo o modelo de Spreitzer (1995) e desse com a variável consequente eficácia gerencial - da empresa. Para testar a hipótese $\mathrm{H}_{4}$ buscaram-se fundamentos nas demais hipóteses deste estudo, para, com auxílio de seus resultados, convalidar esta hipótese que envolve todas as variáveis do estudo:

$\mathrm{H}_{1}$ - $\mathrm{O}$ sistema de recompensa está positivamente relacionado com o empowerment Impacto do Sistema de Recompensa e do Acesso às Informações sobre o Desempenho Individual no... 
psicológico segundo o modelo de Spreitzer (1995) na empresa analisada - não foi aceita devido às dimensões competência $(-0,283)$, autodeterminação $(-0,442)$ e significado $(-0,351)$ terem apresentado coeficientes de regressão negativos. Apenas a dimensão impacto apresentou coeficiente positivo $(0,170)$, porém não significante.

$\mathrm{H}_{2}$ - $\mathrm{O}$ acesso às informações sobre o desempenho individual na empresa pesquisada está positivamente relacionado ao empowerment psicológico de acordo com o modelo proposto por Spreitzer (1995) - foi aceita parcialmente uma vez que a dimensão significado apresentou uma relação positiva $(0,41)$ e significância $(0,04)$ com base no coeficiente de regressão.

$\mathrm{H}_{3^{-}} \mathrm{O}$ empowerment psicológico segundo o modelo de Spreitzer (1995) está positivamente relacionado com a eficácia gerencial na empresa estudada - foi aceita a partir da tendência observada nas informações qualitativas presentes nas entrevistas com os gestores e nas quantitativas oriundas dos indicadores de lucratividade e rentabilidade do período de 2007 a 2010.

Com base no comportamento observado nas três hipóteses anteriores, depreende-se que possa ser parcialmente aceita a hipótese $\mathrm{H}_{4}-\mathrm{O}$ impacto das variáveis sistema de recompensa e acesso às informações sobre o desempenho individual (antecedentes) no empowerment psicológico está positivamente relacionado com a eficácia gerencial (consequente) na empresa analisada.

Estes resultados coadunam parcialmente com a afirmação de Lawler III (2003), ao constatar que o sistema de avaliação de desempenho é mais eficaz quando há ligação com os resultados do sistema de recompensa de uma empresa. Por outro lado, corrobora com os achados de Drake, Wong e Salter (2007), os quais observaram que fornecer feedback sobre o desempenho é susceptível de conduzir a maior motivação dos empregados e produzir um efeito positivo nos resultados da organização.

Na Figura 1 apresenta-se uma síntese das relações hipotéticas encontradas na consecução desta pesquisa e que evidenciam seus achados.

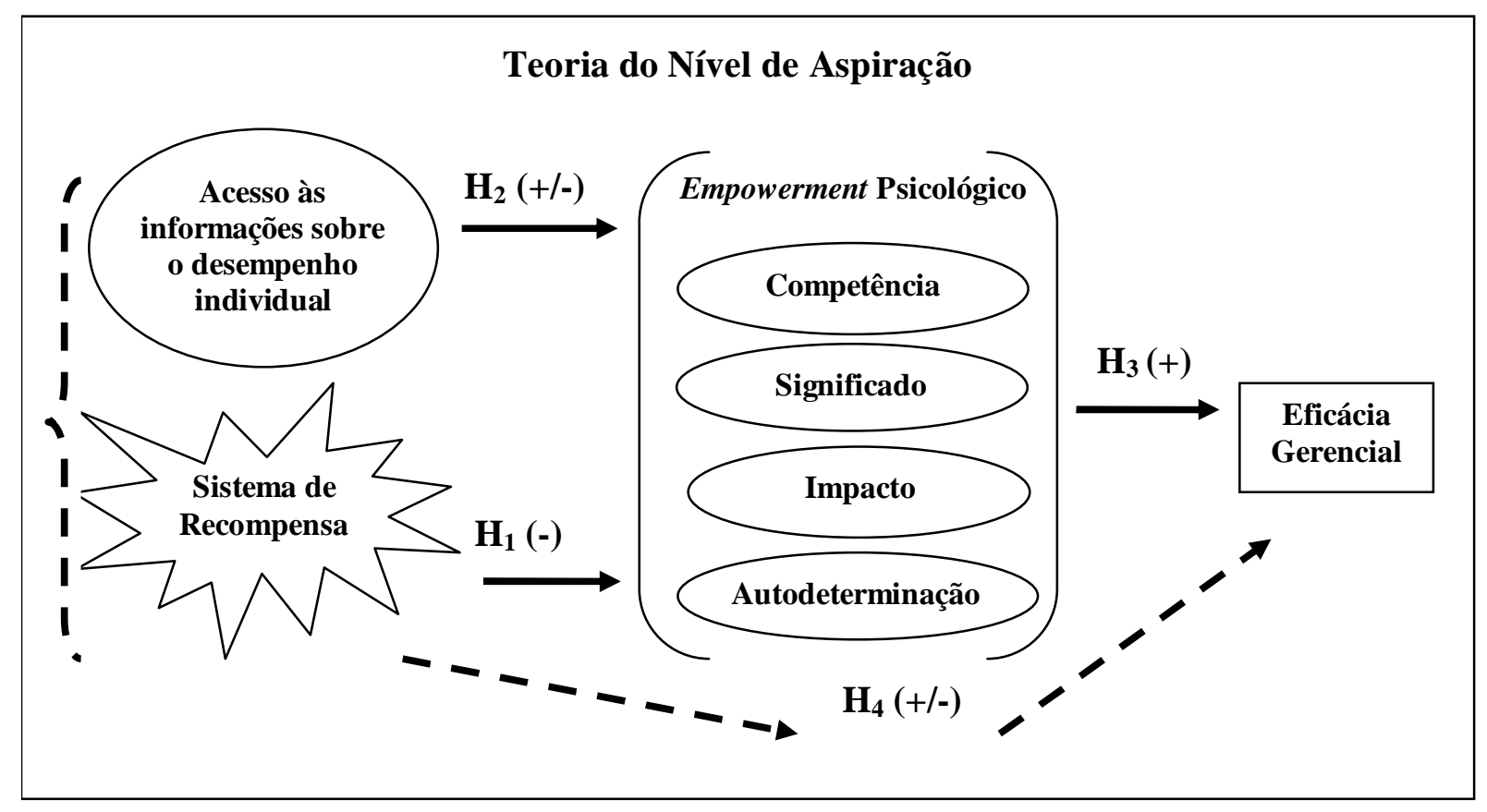

Figura 1 - Síntese das relações hipotéticas encontradas neste estudo 
Depreende-se a partir da confirmação parcial das hipóteses que consubstanciam o estudo, que a utilização do sistema de recompensa e o acesso às informações sobre o desempenho individual dos empregados administrativos e operacionais no empowerment psicológico impactam parcialmente na eficácia gerencial, aferida pelo alcance das metas estabelecidas pelo sistema de recompensa.

Estes achados confirmam a presença dos aspectos psicológicos do capital humano, sobretudo do empowerment psicológico, pois os resultados mostram a confiabilidade das dimensões de competência, impacto, autodeterminação e significado do modelo de Spreitzer (1995) no ambiente organizacional.

Para Spreitzer (1995), o empowerment psicológico encontra-se num estágio embrionário na literatura, tendo em vista o desenvolvimento de sua definição conceitual, medição, validação do constructo e as relações com as variáveis organizacionais. Esta pesquisa validou parte do constructo apresentado por Spreitzer (1995), a partir do conceito apresentado pela autora, e detectou parcialmente sua relação com as variáveis organizacionais - sistema de recompensa, acesso às informações sobre o desempenho individual e eficácia gerencial -, contribuindo, assim, para o preenchimento desta lacuna na literatura.

\section{Conclusões}

O estudo objetivou verificar o impacto das variáveis sistema de recompensa e acesso às informações sobre o desempenho individual (antecedentes) no empowerment psicológico segundo o modelo de Spreitzer (1995), e o seu reflexo na eficácia gerencial (consequente) aferida pelo alcance das metas estabelecidas no sistema de recompensa, em uma empresa multinacional. Pesquisa descritiva com abordagem quantitativa foi realizada em empresa multinacional de origem americana, com uma unidade fabril instalada no Sul do Brasil. O questionário aplicado aos empregados desta unidade fabril foi adaptado de Spreitzer (1995), contendo 24 questões fechadas com escala Likert de sete pontos.

$\mathrm{O}$ estudo fundamentou-se no modelo conceitual de empowerment psicológico de Spreitzer (1995) e da sua rede estrutural utilizaram-se duas variáveis antecedentes (sistema de recompensa e acesso às informações) como inputs e uma variável consequente (eficácia gerencial) como output. O constructo da pesquisa consubstanciou-se de operacionalizações realizadas por Spreitzer (1995) e Lawler III (2003). Na análise dos dados aplicou-se a técnica estatística de regressão linear com a utilização do software Statistical Package for Social Sciences (SPSS).

No que se refere às hipóteses que subsidiaram o estudo, o resultados da pesquisa demonstram que:

A hipótese $\mathrm{H}_{1}-\mathrm{O}$ sistema de recompensa está positivamente relacionado com o empowerment psicológico, segundo o modelo de Spreitzer (1995) - não pode ser aceita, pois a dimensão de significado obteve relação negativa com a variável sistema de recompensa e as demais dimensões não apresentaram significância.

A hipótese $\mathrm{H}_{2}-\mathrm{O}$ acesso às informações sobre o desempenho individual na empresa pesquisada está positivamente relacionado ao empowerment psicológico de acordo com o modelo proposto por Spreitzer (1995) - pode ser aceita parcialmente perante os coeficientes de regressão com valores superiores a 0,10 , mostrando-se não significantes. Apenas a dimensão autodeterminação demonstrou relação positiva e significante, evidenciando sua validade.

A hipótese $\mathrm{H}_{3}-\mathrm{O}$ empowerment psicológico segundo o modelo de Spreitzer (1995) está positivamente relacionado com a eficácia gerencial na empresa estudada - foi aceita a partir do comportamento observado nos indicadores econômico-financeiros de lucratividade e rentabilidade calculados que evidenciam sua evolução ao longo do tempo, cujas informações Impacto do Sistema de Recompensa e do Acesso às Informações sobre o Desempenho Individual no... 
foram reforçadas nas respostas ao instrumento de pesquisa aplicado na empresa.

Ao analisar a relação das variáveis antecedentes - sistema de recompensa e acesso às informações sobre o desempenho individual - com o empowerment psicológico segundo o modelo de Spreitzer (1995) e desse com a variável consequente - eficácia gerencial - na empresa analisada, constatou-se que a hipótese $\mathrm{H}_{4}$ pode ser aceita parcialmente, considerando-se as tendências de comportamento observadas nas hipóteses $\mathrm{H}_{1}, \mathrm{H}_{2}$ e $\mathrm{H}_{3}$.

Conclui-se que o sistema de recompensa e o acesso às informações sobre o desempenho individual dos empregados administrativos e operacionais no empowerment psicológico impactam parcialmente na eficácia gerencial aferida pelo alcance das metas estabelecidas pelo sistema de recompensa da empresa.

Os resultados deste estudo contribuem para a contabilidade gerencial ao apresentar um instrumento de aferição da relação do sistema de recompensa e do acesso às informações sobre o desempenho individual com aspectos comportamentais dos empregados e seu impacto na eficácia gerencial. Também contribuem ao corroborar, ainda que parcialmente, os achados de Spreitzer (1995) e Drake, Wong e Slater (2007). Recomenda-se para futuras pesquisas investigar como o empowerment psicológico influencia na eficácia gerencial da empresa, considerando o cumprimento quantificado das metas, bem como a aplicação do instrumento de pesquisa em outras empresas e sua comparação com os resultados desse estudo.

\section{Referências}

ASSAF NETO, A. Estrutura e análise de balanços: enfoque econômico e financeiro. 6.ed. São Paulo: Atlas, 2001.

ANTHONY, R.N.; GOVINDARAJAN, V. Sistema de controle gerencial. São Paulo: Atlas, 2002.

BERGAMINI, C.W. Psicologia aplicada à administração de empresas: psicologia do comportamento organizacional. 3 ed. São Paulo: Atlas, 1982.

BLANCHARD, K.; CARLOS, J.P.; RANDOLPH, A. As 3 chaves do empowerment: guia prático. São Paulo: Editora Record, 2001.

BORDIN, C.; BARTRAM, T.; CASIMIR, G. The antecedents and consequences of psychological empowerment among Singaporean IT employees. Management Research News, v. 30, n. 1, p. 34-46, 2007.

CONGER, J.A.; KANUNGO, R.N. The empowerment process: integrating theory and Practice. Academy of Management Review, v. 13, n. 3, p. 471-482, 1988.

CICHELERO, M. Medidas de desempenho das empresas e avaliação das pessoas: a contribuição da controladoria. 2004. 95 f. Dissertação (Mestrado Profissional em Economia) Universidade Federal do Rio Grande do Sul, Porto Alegre, 2004.

DIMITRIADES, Z.S. Employee empowerment in the Greek context. International Journal of Manpower, v. 26, n. 1, p. 80-92, 2005.

DIRECTORY OF OPEN ACESS JOURNALS (DOAJ). About. Disponível em: <http://www.doaj.org/doaj?func=loadTempl\&templ=about>. Acesso em: 31 jul. 2010.

DRAKE, A.; WONG, J.; SALTER, S. Empowerment, motivation, and performance: Nascimento, S.; Beuren, I. M. 
examining the impact of feedback and incentives on nonmanagement employees. Behavioral Research in Accounting, v. 19, p. 71-89, 2007.

FREZATTI, F.; ROCHA, W.; NASCIMENTO, A.R.; JUNQUEIRA, E. Controle gerencial: uma abordagem da contabilidade gerencial no contexto econômico, comportamental e sociológico. São Paulo: Atlas, 2009.

GHANI, N.A.A.; HUSSIN, T.A.B.S.B.R.; JUSOFF, K. Antecedents of Psychological Empowerment in the Malaysian Private Higher Education Institutions. International Education Studies. v. 2, n. 3, p. 1-5, 2009.

GRIFFIN, R.W.; MOORHEAD, G. Fundamentos do comportamento organizacional. São Paulo: Ática, 2006.

HAIR JR, J.F.; ANDERSON, R.E.; TATHAM, R.L.; BLACK, W.C. Análise multivariada de dados. 5 ed. Porto Alegre: Bookman, 2005.

HOFSTEDE, G. The universal and the specific in $21^{\text {st }}$-century global management. Organizational Dynamics, v. 28, n. 1, p. 34-44, 1999.

HOLDSWORTH, L.; CARTWRIGHT, S. Empowerment, stress and satisfaction: an exploratory study of a call centre. Leadership \& Organization Development Journal, v. 24, n. 3, p. 131-140, 2003.

LAWLER III, E.E. Reward practices and performance management system effectiveness. Organizational Dynamics, v. 32, n. 4, p. 396-404, 2003.

MARTINS, G.A.; THEÓPHILO, C.R. Metodologia da investigação científica para ciências sociais aplicadas. São Paulo: Atlas, 2007.

MATTHEWS, R.A.; DIAZ, W.M.; COLE, S.G. The organizational empowerment scale. Personnel Review, v. 32, n. 3, p.297-318, 2003.

MENON, S.T. Psychological empowerment: definition, measurement and validation. Canadian Journal of Behavioral Science, v. 31, n. 3, p. 161-164, 1999.

MENON, S.T. Employee empowerment: an integrative psychological approach. Applied Psychology: An International Review, v. 50, n. 1, p. 153-180, 2001.

NASCIMENTO, A.M.; REGINATO, L. Controladoria: um enfoque na eficácia organizacional. 2 ed. São Paulo: Atlas, 2009.

NUNES, A.A. Concessão de opções de ações a funcionários: um problema contábil. BASE Revista de Administração e Contabilidade da Unisinos, v. 5, n. 1, p.32-42, jan./abr., 2008.

PATAH, M.O.R.A.; RADZI, S.M.; ABDULLAH, R.; ADZMY, A.; ZAIN, R.A.; DERANI, $\mathrm{N}$. The influence of psychological empowerment on overall job satisfaction of front office receptionists. International Journal of Business and Management. v.4, n.11, p. 1-10, 2009.

RYAN, R.M.; DECI, E. Intrinsic and extrinsic motivations: classic definitions and new directions. Contemporary Educational Psychology, v. 25, p. 54-67. 2000. 
SIEGALL, M.; GARDNER, S. Contextual factors of psychological empowerment. Personnel Review, v. 29, n. 6, p. 703-722, 2000.

SPREITZER, G. Psychological empowerment instrument. Disponível em: $<\mathrm{http}$ ://webuser.bus.umich.edu/spreitze/empowermentinstrument.pdf $>$. Acesso em: $20 \mathrm{de}$ maio de 2010.

SPREITZER, G. Taking stock: A review of more than twenty years of research on empowerment at work. In.: COOPER, C.; BARLIN, J. (Orgs.). Handbook of organizational behavior. Sage Publications, 2007.

SPREITZER, G. Psychological empowerment in the workplace: dimensions, measurement and validation. Academy of Management Journal, v. 38, n. 5, p.1442-1465, 1995.

THOMAS, K.W.; VELTHOUSE, B.A. Cognitive elements of empowerment: an "interpretive" model of intrinsic task motivation. Academy of Management Review, v. 15, n. 4, p. 666-681, 1990.

VIEIRA, D.P.; PACHECO, R.C.S. Especificações da matriz esforço versus recompensa para organizações do conhecimento. In: ENCONTRO NACIONAL DE ENGENHARIA DE PRODUÇÃO, 24., 2004, Florianópolis. Anais... Florianópolis: ENEGEP. 2004. CD-ROM.

YIN, R.K. Estudo de caso: planejamento e métodos. São Paulo: Bookman, 2003.

ZAPELINI, M.B. Avaliação de empowerment: limites e possibilidades de aplicação na gestão de recursos hídricos no Brasil. In: ENCONTRO DE ADMINISTRAÇÃO PÚBLICA E GOVERNANÇA, 2008, Salvador. Anais... Rio de Janeiro: ANPAD. 2008. CD-ROM. 